\section{Application of the Neutral Zone in Prosthodontics}

Authors: Joseph J. Massad, David R.

Cagna, Charles J. Goodacre, Russell A.

Wicks, Swati A. Ahuja

Publisher:Wiley-Blackwell, UK

Language: English

ISBN: 978-1-1191-5814-1

Edition: 1/e

Publish Year: 2017

Pages: 176, illustrated

Price: $71.50 £$

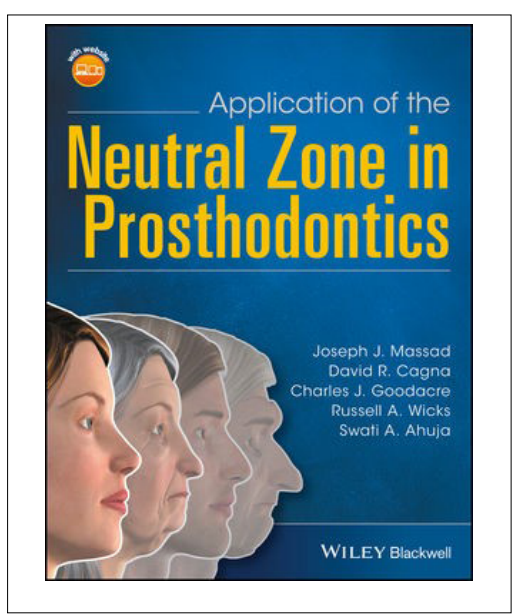

Marian-Vladimi

Constantinescu

DDS, PhD

Holistic Dental \& Medical Institute of Bucharest-ROPOSTURO

Bucharest, Romania

dr.vladimir.constantinescu@gmail.com

The success of the edentulous patients' treatment also involves understanding the importance of the neutral zone in prosthetic dentistry. Professor Joseph J. Massad, together with his colleagues, has sought to provide us with the methods and techniques in which the neutral zone must be incorporated.

The book entitled "Application of the Neutral Zone in Prosthodontics" contains 11 chapters and an index. The main objective of the book is to describe the current procedures in fabricating prostheses that include the neutral zone: implant-supported overdentures, fixed complete dentures and complete dentures.

The authors exemplify the evaluation of edentulous patients, starting with the interview, facial analysis, prosthetic factors, oral characteristics, and then moving on to conditioning the abused tissues and stabilizing the existing prosthesis, seeking re-establishing the orthopedic mandibular position.

There is an innovative, personal technique useful for each type of clinical prosthesis that simplifies fingerprinting, and reduces the number of clinical procedures.

After the production of the definitive models, it continues with the laboratory procedures, the production of record bases, occlusal rims and the mounting of a central bearing device (designed by Massad) indicated in the preparation of the clinical phase of recording the centric relation.

After adjusting the wax occlusal rim in alignment with the facial shape, mount the maxillary cast in the articulator to the transverse horizontal axis and then record the centric relation with the jaw recorder device. A separate chapter covers the recording of the physiological neutral zone for edentulous patients with an impression compound, and for a dentate patient with vinyl polysiloxane (VPS)impression material. Then there follows the selection and arrangement of the anterior teeth, maxillary and mandibular, and then the posterior, mandibular and maxillary teeth, depending on neutral zone registration. There follows the evaluation of wax trial dentures, firstly extra-oral evaluation, the teeth arrangement on the articulator, and then the intra-oral evaluation, esthetics, phonetics, OVD, CR position, and realization of external impressions through appropriate movements to physiologically mold the labial and buccal polished surfaces for one arch at a time.

The treatment is completed by a series of procedures necessary for definitive dentures integration. In the last chapter the book shows a detailed presentation of the use of CAD / CAM technology to record and fabricate neutral zone dentures.

The book is a valuable guide for students, general dentistry residents, prosthodontics, and practitioners who want to understand the neutral zone as a guarantee of the success of a prosthetic treatment.

DOI: https://doi.org/10.25241/stomaeduj.2019.6(1).bookreview.6

The Books Review is drafted in the reviewer's sole wording and illustrates his opinions. 